\title{
La vigencia de los patrones clásicos de cobertura de las catástrofes naturales en la era de Internet ${ }^{1}$
}

\author{
Recibido: 19 de septiembre de 2011 \\ Aceptado: 30 de marzo de 2012 \\ Publicado: 31 de octubre de 2012
}

\author{
Elvira García de Torres \\ egarcia@uch.ceu.es \\ Blanca Nicasio Varea \\ blanca.nicasio@uch.ceu.es \\ Universidad CEU Cardenal Herrera (España)
}

Resumen: En este artículo se presentan los resultados obtenidos tras realizar un análisis comparado del tratamiento periodístico de los desastres naturales en El País y El Mundo durante los años 2009 y 2010, así como indicadores de interés social en Internet. Los objetivos del trabajo, de carácter exploratorio, son descubrir si los valores-noticia clásicos modelan hoy la agenda informativa de los diarios, la agenda global y ciudadana y si el cuaderno de quejas en este campo de especialización tiene vigencia en la era de Internet.

Palabras clave: Desastres naturales, prensa española, agenda-setting, Google, medios sociales.

Abstract: This article presents the results of a comparative analysis of the Press coverage of natural disasters in the world by Spanish newspapers during the years 2009 and 2010 and some prominent indicators of social interest in the Internet. The main goals of this exploratory research are to find out if the classic news values shape altogether the agenda of the mainstream newspapers and the citizens and if the cahier de doléances in this specialized field are also in effect in the Internet age.

Key words: Natural Disasters, Spanish Press, Agenda-Setting, Google, Social Media.

1 Esta investigación se enmarca en el proyecto "Supervivencia del periodismo en la era post-digital. Producción de contenidos en los medios emergentes, consecuencias de la participación ciudadana y evolución de las audiencias", subproyecto CSO2011-29510-C03-02, financiado por el Ministerio de Ciencia e Innovación de España y dirigido por Elvira García de Torres. Asimismo, cuenta con financiación de la Universidad CEU Cardenal Herrera (PRCEU-UCH22/11). 


\section{Introducción}

En los últimos años, el interés de la comunidad internacional por los desastres naturales ha estado marcado por el aumento del riesgo y la vulnerabilidad medioambiental crecientes (aproximadamente el 75\% de la población mundial vive en zonas que han sido afectadas, como mínimo, una vez entre 1980 y el 2000, por algún desastre natural) así como por la actividad de los medios de comunicación, que juegan un papel cada vez mayor en la prevención, mitigación y respuesta en situaciones que reclaman ayuda humanitaria $(c f$. Adam, 1999; Roberts, 1999; Nos Aldás, 2002; O’Heffernan, 1991; Mendiluce, 1997; Brown y Minty, 2008; Cate, 1996 y Gowing, 2001).

El tema, en el seno de la moderna "civilización del riesgo"2 es de gran actualidad e importancia social, porque los efectos de la cobertura pueden ser muy relevantes: "La cobertura ofrecida por los medios condiciona la amplitud de la respuesta dada por la comunidad internacional. Así lo demuestran los niveles, nunca antes vistos, de donaciones alcanzados tras el tsunami en el Sudeste asiático en 2004" (Tristán, 2007: 101).

No solo en los medios tradicionales se hace eco de noticias relacionadas con crisis humanitarias (cf. Kremer, 2007: 36; Jérez, Sampedro y López, 2008: 360; CONGDE, 2007), sino que con el desarrollo de los medios sociales se produce un efecto globalizador, acompañado de cambios en las rutinas de producción, edición y difusión de noticias (Mullaney, 2012).

Así, los vídeos sobre el tsunami, que en 2011 afectó el funcionamiento de la central nuclear de Fukushima (Japón), fueron vistos durante la semana siguiente a la catástrofe 96 millones de veces; pero, además, el tsunami fue en exclusiva el tema central de los veinte vídeos más vistos en YouTube entre el 11 y el 18 de marzo de 2011 y, a medio plazo, entre enero de 2011 y marzo de 2012, se mantuvo como el tema más popular a nivel mundial (Rosenstiel y Mitchell, 2012).

\section{La cobertura periodística de catástrofes naturales: estado de la cuestión}

Los desastres naturales que no se anuncian se enmarcan en la categoría de eventos no programados o "explosiones" (Tuchman, 1983), lo que aumenta la dificultad de la cobertura en un contexto de acomodación a las rutinas noticiosas basadas en previsiones de agenda. En este difícil entorno, la eficiencia de la cobertura varía.

En 1985, tras el seísmo que sufrió Mendoza (Argentina), se difundieron noticias contradictorias, angustiosas y alarmistas. Se produjo una desinformación en forma de rumores que alcanzó las páginas de los diarios, causando alarma y miedo (Bratschi, 1995: 79). Portales (1988) subraya la actuación de los periodistas en 1939, tras el terremoto que devastó Chillán, una localidad situada a 350 kilómetros de Santiago de Chile y que causó más de 5000 víctimas mortales. Los periodistas lograron registrar la información sobre

2 Expresión acuñada a principios de los ochenta tras los sucesos de Seveso (1976) y Three Mile Island (1979) que dio una clara conciencia de una serie de nuevos riesgos a los que se tenían que enfrentar las sociedades actuales. 
el desastre para publicarla al día siguiente y, con el paso de los días, dar información pormenorizada y detallada sobre la tragedia hasta el punto que los locales de los diarios se convirtieron en el centro donde acudir a buscar información sobre familiares desaparecidos. También en 1960, ante la falta de información del Gobierno tras un terremoto que azotó el sur de este país, los periodistas se desplazaron hasta el lugar de los hechos para narrar en primera persona qué estaba sucediendo.

Varios autores defienden, como alternativa a una cobertura tan determinada por la inmediatez, el periodismo preventivo, el periodismo de precisión y el periodismo especializado (Moeller, 2006; Ciancio, 2007). Un ejemplo claro es la investigación clásica del periodista del Miami Herald, Steve Doigt, quien logra demostrar tras el paso del huracán Andrew en Florida, que las edificaciones construidas después del año 1979 no estuvieron sujetas a una exigente normativa de construcción las anteriores. De ahí que tras el paso del ciclón el número de edificios declarados en destrucción total fue dos veces superior que los construidos después de 1979 (Meyer, 2002).

Más recientemente, podemos mencionar al premio Pulitzer Sheri Frink y su reportaje publicado en el diario digital Propublica ("The Deadly Choice at Memorial"). En él, la periodista y médico investiga las prácticas de triaje y las polémicas muertes de varios pacientes en un hospital de Nueva Orleans tras el paso del huracán Katrina. Por otra, el premio ONA que recibió la estación WNYC Radio por “A Snow Storm hits New York. Listeners map the Cleanup" (2011), un mapa ciudadano que incorporó las informaciones brindadas por la audiencia.

En la actualidad, las nuevas herramientas facilitan el trabajo de los periodistas para localizar fuentes ocasionales y expertas. Asimismo, surgen como alternativa a la información convencional entidades y organizaciones no gubernamentales, como Alertnet o ReliefWeb creadas por Reuters y por la OCHA (United Nation Office for the Coordination of Humanitarian Affairs) y la ONG MapAction, que se dedican a la recopilación y transmisión de información sobre crisis humanitarias que necesitan ayuda urgente, así como de crisis olvidadas por los medios (Moeller, 2008).

En la cobertura de desastres, los medios digitales y las redes sociales cobran mayor relevancia por la rápida y efectiva difusión de informaciones importantes, así como por el gran número de usuarios a los que alcanzan con herramientas comunicativas que muestran la magnitud y los efectos de la catástrofe y las principales necesidades de la población afectada. De este modo, se puede facilitar la acción de ayuda humanitaria y de cooperación (Vieweg y otros, 2010; Muralidhraran y otros, 2011; Tinker, Fouse y Curre, 2009; Nagar y otros, 2012; Abbasi y otros, 2012; Mullaney, 2012). Tras el terremoto y posterior tsunami en Japón (2011) se activaron mapas de información abierta que ayudaron a los equipos de rescate a tener una visión más clara de la situación sobre el terreno y a establecer prioridades relacionadas con las necesidades alimentarias, la vivienda y los servicios de saneamiento (Gonzalo, 2011). Los ciudadanos también participan, a través de las redes sociales, aportando datos útiles para la ejecución de los mapas.

Incluso para resolver el problema acuciante de países en vías de desarrollo, con áreas no reflejadas en los mapas, las nuevas herramientas aportan soluciones. Tras el seísmo de Haití, 
OpenStreetMap diseñó mapas actualizados con el objetivo de apoyar a las organizaciones de ayuda a encontrar vías para llegar a las zonas afectadas con fuentes (The National Oceanic and Atmospheric Administration, NOAA; Geo-Eye Google, Banco Mundial, Digital Globe, entre otros) que ofrecían datos con un alto nivel de detalle y que complementaron las informaciones aportadas por los usuarios. Este proyecto, según los autores, demostró la efectividad del crowdsourcing para crear mapas en situaciones de emergencia.

Otras herramientas que favorecen una cobertura más eficaz en términos humanitarios, como se ha puesto de manifiesto en los terremotos de Japón y de Chile, entre otros desastres naturales, es el "Google Person Finder", que informa sobre personas desaparecidas y aporta datos de localización. Después del seísmo de Chile, Google activó "Crisis Response", un portal de información y ayuda al país latinoamericano; y "Map Maker", una aplicación para la construcción de mapas que podía ser utilizada por las ONG y otras organizaciones de ayuda (Gonzalo, 2011).

En situaciones de emergencia, además, las redes sociales canalizan mensajes de solidaridad, promueven campañas de donaciones (Reiersgord, 2011) recogen testimonios de primera mano y pueden convertirse en fuentes de información de lo que está sucediendo (Abbasi y otros, 2012). Son, en definitiva, destinos frecuentes tanto para reunir datos como para difundir información relevante de última hora (Vieweg, 2010). Internet y las redes sociales, por otro lado, dan más oportunidades a los medios tradicionales en la cobertura de catástrofes puesto que ofrecen más espacio para los artículos y la posibilidad de incluir gráficos interactivos y vídeos, entre otros elementos (Friedman, 2011).

En cuanto a los estudios académicos, las conclusiones apuntan reiteradamente a una cobertura escasa y marcada por elementos de proximidad cultural, si bien el debate sigue abierto en torno a los efectos políticos y sociales.

En relación con la noticiabilidad, además de los factores que de forma global se asocian a la selección y edición de noticias internacionales (Galtung y Ruge, 1965), se ha estudiado la incidencia de aquellos que podrían tener mayor peso en este tipo de acontecimientos como el número de víctimas mortales, la cantidad de desplazados, los daños producidos en infraestructuras, la pobreza del país afectado o la cercanía geográfica.

Para algunos, una crisis sólo llegará a los titulares si el país afectado tiene relación política o económica con el país del medio de comunicación (Hens, 2007: 96). Otros autores consideran que el interés existe cuanto más próximo esté la zona afectada geográfica y/o culturalmente (Aguirre, 1999: 213). También se menciona la capacidad de emocionar al público (Nos Aldás, 2002: 21). Ciancio (2007: 10 y 13) afirma que la sobrecarga informativa de los medios hace que sólo incluyan aquellas catástrofes que posean características "espectaculares", lo que significa que los contenidos humanitarios tienen que competir cada vez más entre sí para conseguir visibilidad en los medios.

En referencia al número de víctimas, el informe Western Media Coverage of Humanitarian Disasters (2006) no encuentra una vinculación aparente entre la magnitud del desastre y el interés de los medios. Así, el huracán Katrina es el que dejó el menor número de muertos y el menor número de desplazados, pero fue el que más atención provocó en los medios de 
comunicación de todo el mundo ese año. Moeller (2006) considera que muchas crisis de inimaginables proporciones no han recibido atención por parte de los medios. Otros, sin embargo, opinan que el impacto real de la tragedia, es decir, la cantidad de víctimas hace que se incremente en gran medida el interés periodístico y que, por lo tanto, aumente la cobertura (Van Belle, 2000: 64).

Para Junk y Blatter (2010) existen tres factores determinantes. El primero de ellos es la magnitud de la crisis y, aún más importante, la disponibilidad de imágenes espectaculares que hagan visible el sufrimiento. El segundo se refiere al filtro que controla qué elementos entran a formar parte de la agenda de los medios y qué forma adoptan. Cuánto más liberalizados y con mayor vocación internacional sean los medios será más probable que las crisis reciban atención. Por el contrario, cuando el Gobierno controle los medios menos atención recibirán. Esta hipótesis se deriva del argumento de que un Gobierno utiliza el control para desviar la atención de los problemas que podrían obligarle a participar. El último tiene que ver con la aglomeración de la agenda, es decir, la atención que se presta a las tragedias depende no solo del valor informativo propio sino también del resto de los acontecimientos que estén ocurriendo en ese mismo momento y del valor que posean.

En suma, además de contar con breaking news, imágenes o hechos con rasgos espectaculares, violencia y corrupción; existen otros rasgos que determinan la cobertura como las características políticas, económicas y sociales del país, los vínculos comerciales que mantienen con el país al que pertenece el medio, ser una antigua colonia o que el medio cuente con corresponsales en el lugar, entre otros (Wu, 2000).

En los nuevos medios la respuesta social a los mensajes introduce otro tipo de matices. Según el citado estudio de Rosenstiel y Mitchell (2012) un mes después del tsunami que afectó a Japón (2011), obtuvo gran popularidad un vídeo que mostraba la reunión de una mascota con su dueño (figura 1). Ingresan nuevos parámetros de cobertura en la medida en que testigos, webcams situadas en lugares públicos y organizaciones informativas se constituyen en fuentes de la noticia y el material audiovisual, muchas veces en bruto, ganando protagonismo.

Figura 1: valores noticia y medios sociales.

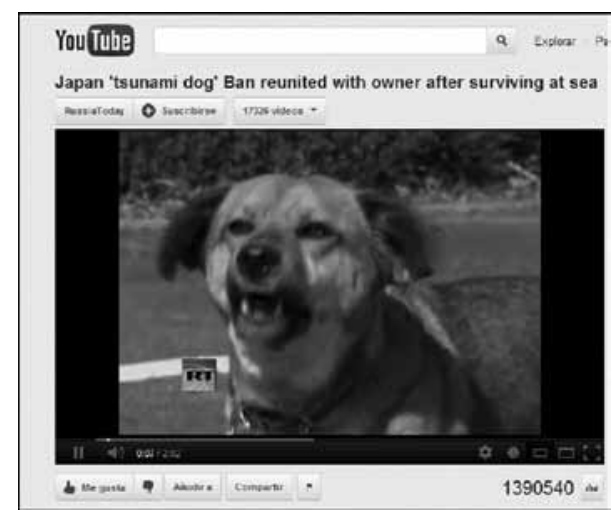

Fuente: Russia Today (Youtube, 2011). 
En cuanto a la influencia, no hay unanimidad en torno a su influencia en la toma de decisiones políticas. Defienden su poder para provocar o legitimar operaciones humanitarias Ciancio (2007), Mendiluce (1997), Sampedro (1997), entre otros. Gidley (2007) asegura que la ayuda internacional para la República Democrática del Congo se triplicó después de que el Comité Internacional de Rescate publicara una investigación sobre la alta mortalidad en el país centroafricano. En otra línea se sitúan los trabajos de Van Belle (2008) y Gowing (2001) que no aprecian tal interrelación de agendas mediática y política.

\section{Objetivos y método aplicado}

El objetivo del presente trabajo consiste en examinar las prioridades de la agenda informativa de los medios de comunicación y su vigencia a la luz de las corrientes globalizadoras y la desaparición de fronteras informativas consecuencia del desarrollo de Internet.

Para llevar a cabo la investigación, acotamos en primer lugar el período de estudio a los años 2009 y 2010, sobre los que es posible recoger evidencias completas. En segundo lugar, examinamos la agenda humanitaria, es decir, el número y características de las catástrofes acaecidas en el período mencionado. Se revisó la agenda global en Google y los índices de tendencias y difusión de noticias en Facebook y Twitter, así como la agenda de la prensa española, concretamente de los diarios El Mundo y El País.

Para obtener información sobre las catástrofes, se han utilizado los informes Annual Disaster Statistical Review: The Numbers and Trends (CRED, 2009 y 2010) que permiten conocer el número de desastres naturales ocurridos durante este período, el número de víctimas, afectados y los daños económicos producidos.

Asimismo, realizamos una búsqueda en Internet de las catástrofes naturales detectadas en Google, a través de una clave de búsqueda que incluye el tipo de catástrofe, el lugar en el que acaece y el año. Asimismo, se anotan los resultados correspondientes a los trending topics y temas más comentados en Twitter y Facebook respectivamente (2009-2010).

El estudio de los textos periodísticos se ha efectuado a través de un análisis de contenido (Bardin, 1986; Krippendorff, 1990; Holst, 1969). Seleccionamos, dada la imposibilidad de abarcar el universo, los diarios El País y El Mundo, los de mayor difusión en el país según la Oficina para la Justificación de la Difusión (OJD), de los que se examinan las secciones "Internacional", "Sociedad", "Ciencia" y "Opinión”. Se han analizado todos los ejemplares correspondientes a los años 2009 y 2010, quedando la muestra conformada por 651 textos (329 publicados en El Mundo y 322 en El País).

En cuanto a la codificación, se establecen parámetros y variables y se dispone de una ficha para procesar los contenidos (datos de registro y presencia o ausencia de variables). Se anotan el nombre del periódico, la fecha de publicación, la sección, el número de página, el titular, la firma, el lugar desde donde se elabora la noticia, el país donde sucede la catástrofe y tipología del desastre. Para determinar el tipo de desastre se utiliza la clasificación elaborada por The Centre for Research on the Epidemiology of Disasters (CRED). En 
cuanto a las variables de análisis, se tienen en cuenta las siguientes: género periodístico, superficie informativa, ubicación, información en portada, página par o impar, fotografía, gráfico y despiece. La superficie informativa se calcula a partir de la medición de una muestra aleatoria de 12 semanas por año.

Planteamos las siguientes hipótesis:

- H1: el tratamiento informativo de las catástrofes naturales en la prensa española es escaso.

- H2: las catástrofes ocurridas en Europa y América Latina reciben mayor cobertura por parte de la prensa española que el resto de catástrofes, independientemente de los indicadores de pérdidas humanas.

- H3: el número de víctimas no es determinante en cuanto a la atención mediática.

- H4: no hay correspondencia con la agenda global pero sí en relación con las tendencias sociales.

\section{Resultados}

Los años examinados se caracterizan por el número de desastres producidos y principalmente el 2010, por la cantidad de víctimas. En el 2009 se contabilizaron 335 desastres naturales y, en 2010, 385 (CRED, 2010 y 2011). En el 2009 hubo 10665 víctimas mortales y más de 119 millones de afectados por desastres naturales. Indonesia fue el país con mayor número de víctimas mortales (1117) y China el que más afectados tuvo, con cerca de 40 millones (CRED, 2010). En el 2010 fueron 385, pero por la gravedad de algunos de ellos las cifras de pérdidas (humanas y materiales) son muy superiores a las del año anterior pues provocaron casi 300000 víctimas mortales y 217 millones de afectados.

América fue el continente más castigado por los desastres naturales debido, especialmente, al terremoto que asoló Haití (enero 2010), en el que murieron más de 222500 personas. Europa contó también con un elevado número de muertes por la ola de calor que afectó a Rusia durante el verano y que causó cerca de 55800 víctimas mortales. En China hubo más de 6400 víctimas debido a varias catástrofes como terremotos e inundaciones. Chile e Indonesia sufrieron graves terremotos que también dejaron un elevado número de muertos. Casi 2000 personas murieron por las inundaciones masivas en Pakistán entre julio y agosto. La ola de frío que sufrió Perú dejó 497 víctimas mortales y los desplazamientos de tierra en Uganda 388 víctimas (CRED, 2011: 2 y 15).

Al examinar los trending topics de la red de microblogging Twitter, encontramos solo una catástrofe en el ranking correspondiente a 2009 y 2010, el terremoto de Haití, que sobresale del nivel de umbral radicalmente, con más de 200000 víctimas, seguida de Rusia con 50000. Vemos que el nivel de umbral es muy alto, pues solo el desastre humanitario en Haití llega a la agenda social global a través de Facebook y Twitter (figura 2), y en un lugar más sobresaliente en el primer caso, pese al carácter más marcadamente informativo que tiene Twitter. 
Figura 2: temas más comentados en Twitter y Facebook (2009-2010).

\begin{tabular}{|c|c|c|c|c|}
\hline RANKING & $\begin{array}{l}\text { TWITTER } \\
\text { (2009) }\end{array}$ & $\begin{array}{l}\text { TWITTER } \\
\text { (2010) }\end{array}$ & $\begin{array}{c}\text { FACEBOOK } \\
\text { (2009) }\end{array}$ & $\begin{array}{l}\text { FACEBOOK } \\
\text { (2010) }\end{array}$ \\
\hline 1 & \#ranelection & FIFA World Cup & $\begin{array}{c}\text { Aplicaciones } \\
\text { FB }\end{array}$ & $\mathrm{HMU}$ \\
\hline 2 & Swine Flu & Inception & FML & $\begin{array}{l}\text { Mundial de } \\
\text { Fútbol }\end{array}$ \\
\hline 3 & Gaza & Haiti Earthquake & Swine flu & Películas \\
\hline 4 & Irán & Vuvuzela & $\begin{array}{l}\text { Celebrity } \\
\text { deaths }\end{array}$ & Ipad/phone \\
\hline 5 & Tehran & Apple iPad & Family & Haití \\
\hline 6 & \#swineflu & $\begin{array}{l}\text { Google } \\
\text { Android }\end{array}$ & Movies & Justin Bieber \\
\hline 7 & AIG & $\begin{array}{l}\text { Justin } \\
\text { Bieber }\end{array}$ & Sports & Juegos \\
\hline 8 & \#uksnow & $\begin{array}{l}\text { Harry Potter \& the } \\
\text { Deathly Hallows }\end{array}$ & Health Care & $\begin{array}{l}\text { Mineros } \\
\text { Chile }\end{array}$ \\
\hline 9 & Earth Hour & Pulpo Paul & FB & Aviones \\
\hline
\end{tabular}

Fuente: elaboración propia, basada en Twitter y Backstrom (2010).

Según datos oficiales de Facebook, las actualizaciones de estado relativas al terremoto de Haití comienzan poco después del terremoto, sobre todo desde República Dominicana, donde se alcanza una tasa de 120 mensajes por minuto, los cuales sumaron 1800 un día después de la catástrofe. La información también surgió de las redes: una mujer de Boston, atrapada con un grupo de 36 compañeros en Haití, utilizó su muro de Facebook para informar a su familia y amigos.

En los medios convencionales analizados (El País y El Mundo) observamos que se cubre un número similar de catástrofes tanto en el 2009 como en el 2010, lo cual resulta sorprendente. Según el Annual Disaster Statistical Review, en el 2009 se produjeron 335 desastres naturales en todo el mundo y en el 2010, 385. El Mundo publicó información sobre 28 catástrofes en el 2009 y 34 en el 2010; El País sobre 25 en el 2009 y 32 en el 2010. 
Por tanto, sólo el $8 \%$ de las catástrofes ocurridas han obtenido espacio en las páginas de estos periódicos, lo que significa que la mayoría de los desastres que ocurrieron en el mundo no formaron parte de la agenda informativa de los medios.

Constatada la opacidad de la mayor parte de las catástrofes que tienen lugar en el mundo, invisibles para los medios examinados, así como la regularidad en cuanto al número de catástrofes sobre las que se informa, encontramos una diferencia que se puede asociar al peso en la agenda humanitaria del elevadísimo número de muertos en el año 2010 frente al 2009. Así, como se aprecia en la figura 3, los medios examinados publicaron 125 textos en el año 2009 y 525 en el año 2010.

Figura 3: textos sobre catástrofes naturales publicados por los diarios analizados (2009-2010).

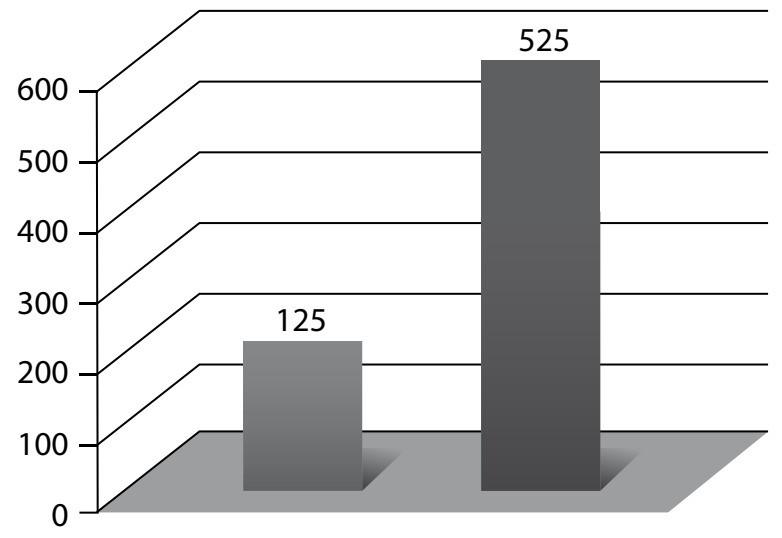

Fuente: elaboración propia.

Los textos analizados se publicaron mayoritariamente en la sección "Internacional" (90\%), seguida de "Opinión" (6\%), "Sociedad" (3\%) y "Ciencia" (1\%). Por lo que se refiere a la autoría, en un $25 \%$ de los casos firma un miembro de la redacción, los corresponsales firmaron el $25 \%$ de las informaciones y los enviados especiales el 20\%. Los textos extraídos de agencias supusieron el $19 \%$ y con "otras firmas" se han correspondido el 11\% de los mensajes. Pese a que la firma de los enviados especiales y corresponsales supone el 45\%, son la noticia y el breve los géneros periodísticos más utilizados (62\%), seguidos de los géneros interpretativos (25\%) y de los de opinión (9\%). En El País, el 74\% de las informaciones contaban con fotografía y en El Mundo el $69 \%$.

Estas diferencias, en cuanto al número de textos por año (lo que redunda en la importancia del número de víctimas) también se detecta en cuanto al número de portadas. En el año 2009, 17 portadas (15 en El País y 2 en El Mundo) incluyeron desastres naturales mientras que en el 2010, la cifra asciende a 71 (43 en El País y 28 en El Mundo). El terremoto de Haití ha sido el desastre que ha contado con más informaciones en portada; seguido del volcán de Islandia. Cabe destacar que a pesar de que dicho volcán no destacó por el número de víctimas (a diferencia de otros desastres como las inundaciones en Pakistán, el terremoto y posterior maremoto de Chile y el terremoto de China), cuenta con un número 
de informaciones en portada superior al del resto de desastres. Llama la atención que en el caso de El Mundo, las inundaciones en Pakistán no cuenten con ninguna información en portada, mientras que la tormenta Xynthia sí. También es llamativo que el huracán Ida (que golpeó El Salvador) apareciera en las portadas de El País.

En el período investigado se han identificado un total de 19 editoriales. Si bien en el año 2009 no se publicó ningún editorial sobre desastres naturales, en el 2010 El País publicó 12 y El Mundo 7. Cabe destacar la diferencia en la superficie informativa (figura 4). Los textos examinados suponen el 1.2\% del total de la superficie informativa en el 2010 en El País y el 1.3\% en El Mundo. Durante el 2009 sólo se registró un $0.2 \%$ en El País y en El Mundo.

Figura 4: superficie informativa dedicada a desastres naturales.

\begin{tabular}{|c|c|c|c|}
\hline DIARIO / Ã̃̃O & TOTAL $\left(\mathbf{c m}^{\mathbf{2}}\right)$ & $\begin{array}{c}\text { CATÁSTROFES } \\
\left(\mathbf{c m}^{\mathbf{2}}\right)\end{array}$ \\
\hline El País (2009) & 11519263.02 & 27998.43 & 0.2 \\
\hline El País (2010) & 10605068.22 & 129714.06 & 1.2 \\
\hline El Mundo (2009) & 10953380.49 & 25546.87 & 0.2 \\
\hline El Mundo (2010) & 8739319.68 & 116167.86 & 1.3 \\
\hline
\end{tabular}

Fuente: elaboración propia.

En el 2010, tres catástrofes sumaron casi el $80 \%$ de la superficie total dedicada a este tipo de hechos. Haití, en correspondencia con la agenda social, ocupó más del 58\% de la superficie informativa en El Mundo y más del $51 \%$ en El País. Representa la catástrofe de Haití, por tanto, más de la mitad de la superficie informativa dedicada a desastres naturales en todo el año.

Una catástrofe sin pérdidas humanas ocupó el segundo lugar: la erupción del volcán islandés Eyjafjalla, con cerca del $12 \%$ de la superficie en El Mundo y del 15\% en El País. Las demás catástrofes (31 en El Mundo y 29 en El País) se repartieron el 20\% restante de la superficie orientada a estos temas.

Asimismo, en el año 2009, tanto El Mundo como El País dedicaron casi el 60\% del total de la superficie informativa sobre catástrofes naturales a los siguientes acontecimientos. El terremoto que golpeó la ciudad italiana L'Aquila fue el que más superficie ocupó en los dos diarios; seguido del terremoto y posterior tsunami que azotó las costas indonesias en el verano de 2009.

Con datos del 2010, se observa que el número de víctimas no implica mayor cobertura, con la excepción del seísmo de Haití. Rusia, que se sitúa como la segunda catástrofe con mayor número de fallecidos, ocupa el cuarto puesto y supone el $3.01 \%$ de la superficie total de $E l$ 
Mundo y el $4.74 \%$ de El País. Por otro lado, China, con casi 3000 víctimas mortales, no llega al $1 \%$ de la superficie de El Mundo y sólo supone el $1.22 \%$ de El País.

Las inundaciones en este mismo país, que dejaron casi 1700 víctimas, ocuparon el $0.13 \%$ de la superficie de El Mundo y el $0.44 \%$ de El País. Las inundaciones producidas en Pakistán (que dejaron casi 2000 víctimas) ocuparon el 6\% de la superficie de El Mundo y el $7 \%$ de El País. Es más, la superficie destinada a Pakistán fue menor respecto a otras catástrofes con un número inferior de víctimas mortales como el terremoto que golpeó Chile. Asimismo, otros hechos como la tormenta Xynthia (Francia), la ola de frío que golpeó Europa, las lluvias y el desplazamiento de tierras en Madeira (Portugal), dejaron un número de víctimas mucho menor pero tuvieron una cobertura mayor.

En 2009, el tifón Morakot (que dejó 664 víctimas mortales) ocupó casi el 5\% de la superficie informativa de El Mundo y casi el 3\% de El País. La tormenta Ketsana (que afectó Filipinas, Vietnam, Camboya y Laos con un saldo de 716 víctimas) ocupó el $0.08 \%$ en El Mundo y el 0,49\% en El País. Las inundaciones en la India (con más de 900 víctimas) no aparecieron en El País. En El Mundo apenas se le dedicó el $0.7 \%$ de la superficie total. A estos datos, podemos añadir que catástrofes como el tifón Pepeng (Filipinas, China y Vietnam) y el ciclón Aila (India, Bangladesh y Bhutan), que dejaron 542 y 298 víctimas respectivamente, no aparecieron en ninguno de los periódicos mencionados. Otras, sin embargo, con un menos número de víctimas (terremotos en México y Costa Rica, el temporal de lluvias en Sicilia o la ola de frío en Europa del norte) recibieron cobertura.

En la figura 5 se presentan los resultados organizados según la atención periodística (descendente), el número de víctimas (descendente) y la distancia geográfica (ascendente). Hemos determinado el índice de atención periodística (IAP) a partir de la suma de cuatro indicadores en los dos diarios: a) publicación en portada, b) editoriales, c) firma y d) publicación de fotografía, de manera que el índice de atención periodística más alto corresponde al 8, la suma de los cuatro indicadores en los dos diarios analizados.

La distancia geográfica se toma en relación con Madrid (España). Además de estos datos, se incluyen los resultados obtenidos al realizar la búsqueda en Google de las catástrofes examinadas y el porcentaje de la correspondencia real de los resultados de la búsqueda en los cien primeros resultados (cuarta columna). 
Figura 5: resultados globales.

\begin{tabular}{|c|c|c|c|c|c|c|}
\hline $\begin{array}{l}\text { CATÁSTROFE Y } \\
\text { AÑNO }\end{array}$ & $\begin{array}{l}\text { VÍCTIMAS } \\
\text { MORTALES }\end{array}$ & GOOGLE & $\% 100$ & IAP & CM2 & KM \\
\hline $\begin{array}{l}\text { Terremoto Haití } \\
\text { (2010) }\end{array}$ & 222641 & 3910000 & $100 \%$ & 8 & $\begin{array}{l}\text { EP: } 52.11 \% \\
\text { EM: } 58.31 \%\end{array}$ & 6895 \\
\hline $\begin{array}{l}\text { Incendios Rusia } \\
\text { (2010) }\end{array}$ & 55844 & 1350000 & $99 \%$ & 8 & $\begin{array}{l}\text { EP: } 4.74 \% \\
\text { EM: } 3.01 \%\end{array}$ & 3435 \\
\hline $\begin{array}{l}\text { Terremoto Chile } \\
\text { (2010) }\end{array}$ & 562 & 26000000 & $100 \%$ & 8 & $\begin{array}{l}\text { EP: } 10.43 \% \\
\text { EM: } 9.11 \%\end{array}$ & 10707 \\
\hline $\begin{array}{l}\text { Tormenta Xynthia } \\
\text { (Europa, 2010) }\end{array}$ & 57 & 24700 & $100 \%$ & 7 & $\begin{array}{l}\text { EP: } 1.08 \% \\
\text { EM: } 1.19 \%\end{array}$ & 1052 \\
\hline $\begin{array}{c}\text { Terremoto China } \\
\text { (2010) }\end{array}$ & 2968 & 6190000 & $100 \%$ & 6 & $\begin{array}{l}\text { EP: } 1.22 \% \\
\text { EM: } 0.44 \%\end{array}$ & 9239 \\
\hline $\begin{array}{l}\text { Inundación } \\
\text { India (2010) }\end{array}$ & 1405 & 1490000 & $100 \%$ & 6 & $\begin{array}{l}\text { EP: } 0.95 \% \\
\text { EM: } 2.23 \%\end{array}$ & 7301 \\
\hline $\begin{array}{l}\text { Terremoto / Tsunami } \\
\text { Indonesia (2009) }\end{array}$ & 1117 & 1900000 & $90 \%$ & 6 & $\begin{array}{l}\text { EP: } 20.34 \% \\
\text { EM: } 11.70 \%\end{array}$ & 12219 \\
\hline $\begin{array}{l}\text { Terremoto L'Aquila } \\
\text { (Italia, 2009) }\end{array}$ & 295 & 3270000 & $100 \%$ & 6 & $\begin{array}{l}\text { EP: } 23.62 \% \\
\text { EM: } 30.28 \%\end{array}$ & 1442 \\
\hline $\begin{array}{c}\text { Incendio Australia } \\
\text { (2009) }\end{array}$ & 173 & 2830000 & $99 \%$ & 6 & $\begin{array}{l}\text { EP: } 5.13 \% \\
\text { EM: } 6.23 \%\end{array}$ & 17603 \\
\hline $\begin{array}{c}\text { Inundación Pakistán } \\
\text { (2010) }\end{array}$ & 2186 & 1040000 & $100 \%$ & 5 & $\begin{array}{l}\text { EP: } 7.20 \% \\
\text { EM: } 6.06 \%\end{array}$ & 6651 \\
\hline $\begin{array}{c}\text { Huracán Ida } \\
\text { (El Salvador, 2009) }\end{array}$ & 281 & 293000 & $100 \%$ & 5 & $\begin{array}{l}\text { EP: } 2.21 \% \\
\text { EM: } 1.05 \%\end{array}$ & 8587 \\
\hline $\begin{array}{c}\text { Incendios Grecia } \\
\text { (2009) }\end{array}$ & 0 & 3410000 & $92 \%$ & 5 & $\begin{array}{l}\text { EP: } 8.91 \% \\
\text { EM: } 6.29 \%\end{array}$ & 2374 \\
\hline $\begin{array}{l}\text { Lluvias Madeira } \\
\text { (Portugal, 2010) }\end{array}$ & 42 & 188000 & $99 \%$ & 4 & $\begin{array}{l}\text { EP: } 1.41 \% \\
\text { EM: } 0.78 \%\end{array}$ & 1455 \\
\hline $\begin{array}{c}\text { Incendios Israel } \\
\text { (2010) }\end{array}$ & 40 & 3450000 & $100 \%$ & 4 & $\begin{array}{l}\text { EP: } 0.46 \% \\
\text { EM: } 1.13 \%\end{array}$ & 3551 \\
\hline $\begin{array}{l}\text { Lluvias México } \\
\text { (2009) }\end{array}$ & 30 & 543000 & $56 \%$ & 4 & $\begin{array}{l}\text { EP: } 0.29 \% \\
\text { EM: } 0.21 \%\end{array}$ & 9101 \\
\hline $\begin{array}{c}\text { Temporal Sicilia } \\
\text { (2009) }\end{array}$ & 22 & 386000 & $63 \%$ & 4 & $\begin{array}{l}\text { EP: } 0.62 \% \\
\text { EM: } 3.19 \%\end{array}$ & 1492 \\
\hline
\end{tabular}




\begin{tabular}{|c|c|c|c|c|c|c|}
\hline $\begin{array}{l}\text { Ola de frío Europa } \\
\text { (2009) }\end{array}$ & 20 (más) & 1280000 & $92 \% 4$ & 4 & $\begin{array}{l}\text { EP: } 2.55 \% \\
\text { EM: } 9.10 \%\end{array}$ & 1875 \\
\hline $\begin{array}{c}\text { Deslizamiento } \\
\text { de tierras México } \\
\text { (2010) }\end{array}$ & 11 & 973000 & $74 \%$ & 4 & $\begin{array}{l}\text { EP: } 0.21 \% \\
\text { EM: } 0.60 \%\end{array}$ & 9101 \\
\hline $\begin{array}{l}\text { Erupción volcán } \\
\text { Islandia (2010) }\end{array}$ & 0 & 518000 & $100 \%$ & 4 & $\begin{array}{l}\text { EP: } 14.46 \% \\
\text { EM: } 11.79 \%\end{array}$ & 2890 \\
\hline $\begin{array}{l}\text { Sequía Suráfrica y } \\
\text { Etiopía (2009) }\end{array}$ & 0 & 460000 & $63 \%$ & 4 & $\begin{array}{l}\text { EP: - } \\
\text { EM: } 1.19 \%\end{array}$ & 5735 \\
\hline $\begin{array}{c}\text { Inundación Zambia } \\
\text { (2009) }\end{array}$ & 0 & 1190000 & $86 \%$ & 4 & $\begin{array}{l}\text { EP: - } \\
\text { EM: } 0.68 \%\end{array}$ & 7037 \\
\hline $\begin{array}{l}\text { Lluvias China } \\
\text { (2010) }\end{array}$ & 1765 & 4010000 & $82 \%$ & 3 & $\begin{array}{l}\text { EP: } 0.47 \% \\
\text { EM: } 0.13 \%\end{array}$ & 9239 \\
\hline $\begin{array}{l}\text { Lluvias Brasil } \\
\text { (2010) }\end{array}$ & 153 & 3840000 & $97 \%$ & 3 & $\begin{array}{l}\text { EP: } 0.80 \% \\
\text { EM: } 0.17 \%\end{array}$ & 7731 \\
\hline $\begin{array}{c}\text { Inundación Francia } \\
\text { (2010) }\end{array}$ & 25 & 2610000 & $93 \%$ & 3 & $\begin{array}{l}\text { EP: } 0.24 \% \\
\text { EM: } 0.02 \%\end{array}$ & 1052 \\
\hline $\begin{array}{c}\text { Inundación } \\
\text { República Checa } \\
\text { (2009) }\end{array}$ & 12 & 1260000 & $84 \%$ & 3 & $\begin{array}{l}\text { EP: } 0.48 \% \\
\text { EM: } 0.43 \%\end{array}$ & 1779 \\
\hline $\begin{array}{l}\text { Incendio Portugal } \\
\text { (2010) }\end{array}$ & 0 & 1940000 & $98 \%$ & 3 & $\begin{array}{l}\text { EP: } 0.79 \% \\
\text { EM: } 0.21 \%\end{array}$ & 504 \\
\hline $\begin{array}{c}\text { Sequía Magadascar } \\
\text { (2009) }\end{array}$ & 0 & 979000 & $72 \%$ & 3 & $\begin{array}{l}\text { EP: } 3.07 \% \\
\text { EM: - }\end{array}$ & 8467 \\
\hline $\begin{array}{c}\text { Inundaciones } \\
\text { Bangladesh (2009) }\end{array}$ & 0 & 47100 & $97 \%$ & 3 & $\begin{array}{l}\text { EP: } 3.07 \% \\
\text { EM: - }\end{array}$ & 8680 \\
\hline $\begin{array}{l}\text { Sequía Argentina } \\
\text { (2009) }\end{array}$ & 0 & 2630000 & $92 \%$ & 3 & $\begin{array}{l}\text { EP: } 6.30 \% \\
\text { EM: - }\end{array}$ & 10053 \\
\hline $\begin{array}{c}\text { Tifón Morakot } \\
\text { (China y Taiwán, } \\
\text { 2009) }\end{array}$ & 664 & 33100 & $100 \%$ & 2 & $\begin{array}{l}\text { EP: } 2.93 \% \\
\text { EM: } 4.98 \%\end{array}$ & 9239 \\
\hline $\begin{array}{l}\text { Erupción Merapi } \\
\text { (Indonesia, 2010) }\end{array}$ & 240 & 200000 & $100 \%$ & 2 & $\begin{array}{l}\text { EP: } 0.42 \% \\
\text { EM: } 0.45 \%\end{array}$ & 12219 \\
\hline $\begin{array}{c}\text { Tormenta Agatha } \\
\text { (Guatemala, 2010) }\end{array}$ & 180 & 848000 & $100 \%$ & 2 & $\begin{array}{l}\text { EP: } 0.25 \% \\
\text { EM: } 0.32 \%\end{array}$ & 8703 \\
\hline $\begin{array}{l}\text { Terremoto Nueva } \\
\text { Zelanda (2010) }\end{array}$ & 100 & 738000 & $100 \%$ & 2 & $\begin{array}{l}\text { EP: } 0.20 \% \\
\text { EM: } 0.18 \%\end{array}$ & 19620 \\
\hline
\end{tabular}




\begin{tabular}{|c|c|c|c|c|c|c|}
\hline $\begin{array}{l}\text { Inundación Turquía } \\
\text { (2009) }\end{array}$ & 31 & 622000 & $96 \%$ & 2 & $\begin{array}{l}\text { EP: } 1.16 \% \\
\text { EM: } 1.47 \%\end{array}$ & 2742 \\
\hline $\begin{array}{c}\text { Ola de frío Europa } \\
\text { (2010) }\end{array}$ & 28 & 3840000 & $71 \%$ & 2 & $\begin{array}{l}\text { EP: } 0.50 \% \\
\text { EM: } 0.87 \%\end{array}$ & 1875 \\
\hline $\begin{array}{l}\text { Incendio Los } \\
\text { Ángeles (Estados } \\
\text { Unidos, 2009) }\end{array}$ & 4 & 3090000 & $90 \%$ & 2 & $\begin{array}{l}\text { EP: } 0.11 \% \\
\text { EM: } 1.39 \%\end{array}$ & 9371 \\
\hline $\begin{array}{l}\text { Tormenta Bonnie } \\
\text { (México, 2010) }\end{array}$ & 2 & 253000 & $100 \%$ & 2 & $\begin{array}{l}\text { EP: } 0.17 \% \\
\text { EM: - }\end{array}$ & 9101 \\
\hline $\begin{array}{l}\text { Ola de frío Estados } \\
\text { Unidos (2010) }\end{array}$ & 0 & 2010000 & $85 \%$ & 2 & $\begin{array}{l}\text { EP: } 0.30 \% \\
\text { EM: } 0.28 \%\end{array}$ & 6092 \\
\hline $\begin{array}{l}\text { Sequía Estados } \\
\text { Unidos (2009) }\end{array}$ & 0 & 2660000 & $47 \%$ & 2 & $\begin{array}{l}\text { EP: } 2.38 \% \\
\text { EM: - }\end{array}$ & 6092 \\
\hline $\begin{array}{l}\text { Sequía India } \\
\text { (2009) }\end{array}$ & 0 & 1190000 & $97 \%$ & 2 & $\begin{array}{l}\text { EP: - } \\
\text { EM: } 3.34 \%\end{array}$ & 7301 \\
\hline $\begin{array}{l}\text { Sequía Amazonas } \\
\text { (Brasil, 2009) }\end{array}$ & 0 & 3760000 & $100 \%$ & 2 & $\begin{array}{l}\text { EP: } 3.07 \% \\
\text { EM: - }\end{array}$ & 7492 \\
\hline $\begin{array}{l}\text { Sequía Amazonas } \\
\text { (Brasil, 2010) }\end{array}$ & 0 & 186000 & $97 \%$ & 2 & $\begin{array}{l}\text { EP: - } \\
\text { EM: } 0.36 \%\end{array}$ & 7731 \\
\hline $\begin{array}{l}\text { Sequía Himalaya } \\
\text { (2009) }\end{array}$ & 0 & 491000 & $89 \%$ & 2 & $\begin{array}{l}\text { EP: } 3.07 \% \\
\text { EM: - }\end{array}$ & 7989 \\
\hline $\begin{array}{l}\text { Volcán Redoubt } \\
\text { (Alaska, 2009) }\end{array}$ & 0 & 19300 & $100 \%$ & 2 & $\begin{array}{l}\text { EP: } 1.11 \% \\
\text { EM: - }\end{array}$ & 8317 \\
\hline $\begin{array}{l}\text { Terremoto México } \\
\text { (2009) }\end{array}$ & 0 & 9650000 & $82 \%$ & 2 & $\begin{array}{l}\text { EP: } 1.83 \% \\
\text { EM: - }\end{array}$ & 9072 \\
\hline $\begin{array}{c}\text { Inundaciones China } \\
\text { (2009) }\end{array}$ & 0 & 2670000 & $68 \%$ & 2 & $\begin{array}{l}\text { EP: } 3.07 \% \\
\text { EM: - }\end{array}$ & 9239 \\
\hline $\begin{array}{l}\text { Sequía Chad } \\
\text { (2010) }\end{array}$ & $\begin{array}{c}\text { Millones } \\
\text { de afectados }\end{array}$ & 1010000 & $99 \%$ & 2 & $\begin{array}{l}\text { EP: - } \\
\text { EM: } 0.39 \%\end{array}$ & 3653 \\
\hline $\begin{array}{c}\text { Inundaciones India } \\
\text { (2009) }\end{array}$ & 992 & 1220000 & $79 \%$ & 1 & $\begin{array}{l}\text { EP: - } \\
\text { EM: } 0.74 \%\end{array}$ & 7301 \\
\hline $\begin{array}{c}\text { Tormenta Kelsana / } \\
\text { Inundación Filipinas } \\
\text { (2009) }\end{array}$ & 716 & 994000 & $100 \%$ & 1 & $\begin{array}{l}\text { EP: } 0.49 \% \\
\text { EM: } 0.08 \%\end{array}$ & 11675 \\
\hline $\begin{array}{c}\text { Tsunami Indonesia } \\
\text { (2010) }\end{array}$ & 530 & 19200000 & $99 \%$ & $\begin{array}{l}\text { EP: } 1 \\
\text { EM: - }\end{array}$ & $\begin{array}{l}\text { EP: } 0.18 \% \\
\text { EM: } 0.02 \%\end{array}$ & 12219 \\
\hline
\end{tabular}




\begin{tabular}{|c|c|c|c|c|c|c|}
\hline $\begin{array}{l}\text { Inundación Brasil } \\
\text { (2010) }\end{array}$ & 213 & 2720000 & $83 \%$ & 1 & $\begin{array}{l}\text { EP: } 0.09 \% \\
\text { EM: } 0.18 \%\end{array}$ & 7731 \\
\hline $\begin{array}{l}\text { Alud de tierra } \\
\text { (colombia, 2010) }\end{array}$ & 74 & 330000 & $98 \%$ & 1 & $\begin{array}{l}\text { EP: - } \\
\text { EM: } 0.13 \%\end{array}$ & 8029 \\
\hline $\begin{array}{l}\text { Lluvias Afganistán } \\
\text { (2010) }\end{array}$ & 28 & 236000 & $36 \%$ & 1 & $\begin{array}{l}\text { EP: - } \\
\text { EM: } 0.20 \%\end{array}$ & 6281 \\
\hline $\begin{array}{c}\text { Tifón Megi } \\
\text { (Filipinas, 2010) }\end{array}$ & 26 & 201000 & $100 \%$ & 1 & $\begin{array}{l}\text { EP: } 0.09 \% \\
\text { EM: - }\end{array}$ & 11675 \\
\hline $\begin{array}{c}\text { Terremoto } \\
\text { Afganistán (2009) }\end{array}$ & 22 & 1190000 & $76 \%$ & 1 & $\begin{array}{l}\text { EP: - } \\
\text { EM: } 0.09 \%\end{array}$ & 6281 \\
\hline $\begin{array}{l}\text { Huracán Alex } \\
\text { (México, 2010) }\end{array}$ & 17 & 1480000 & $99 \%$ & 1 & $\begin{array}{l}\text { EP: - } \\
\text { EM: } 0.20 \%\end{array}$ & 9101 \\
\hline $\begin{array}{l}\text { Temporal de lluvias } \\
\text { (Argentina, 2010) }\end{array}$ & 17 & 1900000 & $57 \%$ & 1 & $\begin{array}{l}\text { EP: - } \\
\text { EM: } 0.17 \%\end{array}$ & 10053 \\
\hline Alud Austria (2009) & 6 & 942000 & $43 \%$ & 1 & $\begin{array}{l}\text { EP: } 0.24 \% \\
\text { EM: } 1.01 \%\end{array}$ & 1815 \\
\hline $\begin{array}{l}\text { Tormenta de granizo } \\
\text { (Australia, 2010) }\end{array}$ & 5 & 122000 & $91 \%$ & 1 & $\begin{array}{l}\text { EP: - } \\
\text { EM: } 0.19 \%\end{array}$ & 17603 \\
\hline $\begin{array}{c}\text { Terremoto México } \\
\text { (2010) }\end{array}$ & 2 & 18400000 & $98 \%$ & 1 & $\begin{array}{l}\text { EP: } 0.02 \% \\
\text { EM: } 0.17 \%\end{array}$ & 9101 \\
\hline $\begin{array}{l}\text { Sequía África } \\
\text { Occidental }\end{array}$ & 0 & 68000 & $63 \%$ & 1 & $\begin{array}{l}\text { EP: - } \\
\text { EM: } 0.59 \%\end{array}$ & 6197 \\
\hline $\begin{array}{c}\text { Erupción } \\
\text { Tungurahua } \\
\text { (Ecuador, 2010) }\end{array}$ & 0 & 218000 & $100 \%$ & 1 & $\begin{array}{l}\text { EP: } 0.11 \% \\
\text { EM: - }\end{array}$ & 8755 \\
\hline $\begin{array}{l}\text { Inundaciones } \\
\text { Maldivas (2009) }\end{array}$ & 0 & 20500 & $100 \%$ & 1 & $\begin{array}{l}\text { EP: - } \\
\text { EM: } 0.59 \%\end{array}$ & 8952 \\
\hline $\begin{array}{l}\text { Terremoto Costa } \\
\text { Rica (2009) }\end{array}$ & SD & 2790000 & $100 \%$ & 1 & $\begin{array}{l}\text { EP: - } \\
\text { EM: } 1.03 \%\end{array}$ & 8489 \\
\hline $\begin{array}{l}\text { Tifón Pepeng } \\
\text { (Filipinas, 2009) }\end{array}$ & 542 & 979 & $97 \%$ & 0 & $\begin{array}{l}\text { EP: - } \\
\text { EM: } 0.14 \%\end{array}$ & 11675 \\
\hline $\begin{array}{l}\text { Alud de tierra } \\
\text { Uganda (2010) }\end{array}$ & 388 & 53900 & $100 \%$ & 0 & $\begin{array}{l}\text { EP: } 0.02 \% \\
\text { EM: - }\end{array}$ & 5776 \\
\hline $\begin{array}{l}\text { Inundaciones Arabia } \\
\text { Saudita (2009) }\end{array}$ & 103 & 158000 & $71 \%$ & 0 & $\begin{array}{l}\text { EP: - } \\
\text { EM: } 0.18 \%\end{array}$ & 4538 \\
\hline Lluvias Brasil (2010) & 79 & 3840000 & $97 \%$ & 0 & $\begin{array}{l}\text { EP: - } \\
\text { EM: - }\end{array}$ & 7731 \\
\hline
\end{tabular}




\begin{tabular}{|c|c|c|c|c|c|c|}
\hline $\begin{array}{c}\text { Terremoto Turquía } \\
(2010)\end{array}$ & 57 & 2280000 & $100 \%$ & 0 & $\begin{array}{l}\text { EP: } 0.34 \% \\
\text { EM: }-\end{array}$ & 2742 \\
\hline $\begin{array}{c}\text { Inundación Polonia } \\
(2010)\end{array}$ & 21 & 648000 & $100 \%$ & 0 & $\begin{array}{l}\text { EP: } 0.02 \% \\
\text { EM: } 0.03 \%\end{array}$ & 2299 \\
\hline $\begin{array}{c}\text { Inundación Australia } \\
(2010)\end{array}$ & 20 & 1220000 & $99 \%$ & 0 & $\begin{array}{l}\text { EP: }- \\
\text { EM: } 0.02 \%\end{array}$ & 17603 \\
\hline $\begin{array}{c}\text { Inundaciones } \\
\text { Georgia (Estados } \\
\text { Unidos, 2009) }\end{array}$ & 9 & 996000 & $81 \%$ & 0 & $\begin{array}{l}\text { EP: }- \\
\text { EM: } 0.19 \%\end{array}$ & 6945 \\
\hline $\begin{array}{c}\text { Terremoto Japón } \\
\text { (2009) }\end{array}$ & 1 & 4800000 & $43 \%$ & 0 & $\begin{array}{l}\text { EP: }- \\
\text { EM: } 0.10 \%\end{array}$ & 10770 \\
\hline
\end{tabular}

Fuente: elaboración propia.

El interés mundial por el terremoto de Haití tiene una clara correspondencia con el tratamiento informativo de la prensa española, ya que supone el $50 \%$ de la cobertura de los diarios, pero vemos el peso del factor cultural en el tratamiento del terremoto chileno, que alcanza un IAP similar. Es interesante también la visibilidad que otorga la portada del diario, más abierta a las catástrofes que el trending topic global de Twitter o los estados en Facebook. Obviamente, los desastres naturales no están presentes de forma prioritaria en la agenda social, puesto que la catástrofe rusa (que registró 55000 muertos) no entró en la lista de temas más comentados.

En cuanto a los resultados obtenidos en Google, relativos a la memoria de Internet en relación con las catástrofes, consideramos que pese a su interés no son concluyentes y que sería preciso establecer un índice superior de fiabilidad de las búsquedas al empleado en este estudio.

\section{Conclusiones}

Los resultados avalan la vigencia de viejos patrones informativos en la cobertura de catástrofes naturales, la existencia de un alto número de estos que escapan a la atención mediática y mayor visibilidad de aquellos en los que se dan las variables de proximidad cultural y/o geográfica, confirmándose las hipótesis planteadas.

El número de víctimas, cuando sobrepasa de forma extraordinaria el nivel de umbral (como es el caso de Haití), tiene un reflejo en la cobertura informativa en cuanto a tratamiento editorial, espacio y portadas. Sin embargo, esto no se cumple de forma universal. Basta citar el terremoto que sufrió Indonesia (2009), las inundaciones en la India (mil víctimas) o la tormenta Ketsana (700 víctimas) que no recibieron la atención que suscitó el terremoto en L'Aquila (Italia) donde fallecieron 295. La erupción del volcán Eyjafjalla (Islandia), sin víctimas mortales, recibió una gran cobertura mediática debido a que bloqueó el tráfico aéreo europeo con graves consecuencias económicas. 
La superficie informativa que se dedicó a las catástrofes fue, en términos generales y relativos, pobre, porque con la excepción de grandes catástrofes que atrapan la atención internacional de forma extraordinaria, compiten un número muy reducido del total de catástrofes que tienen lugar en el mundo, menos del 10\%, por este limitado espacio.

La proximidad cultural también queda validada como criterio noticioso. Así, por ejemplo, vemos que desastres ocurridos en países de América Latina o Europa han recibido, en general, más superficie que otros que han contado con un número mayor de víctimas, como el terremoto de L'Aquila frente a las inundaciones en India, la tormenta tropical Ketsana o los tifones Morakot y Pepeng que recibieron una cobertura menor, además de contar con corresponsales y enviados especiales.

Haití ha sido con diferencia la catástrofe con más peso en la agenda mediática de los años 2009 y 2010, así como trending topic de Twitter y uno de los temas más comentados en Facebook. Pero han sido precisas más de 200000 víctimas mortales para que el terrible acontecimiento entrara en la agenda social global. Los resultados obtenidos en Google no refuerzan, sin embargo, la apreciación social y mediática de su valor de umbral.

Si la cobertura se proyecta sobre la ayuda humanitaria y la conversación social influye en la agenda de los medios, las consecuencias de la revolución 2.0 pueden tener alcance económico y social importante en las zonas afectadas por catástrofes naturales. Por ese motivo, es del mayor interés, en cuanto a la investigación del futuro, no solo profundizar en la relación entre los indicadores de ayuda humanitaria y la cobertura mediática, sino estudiar su interrelación con la agenda ciudadana así como con la huella que dejan las catástrofes en Internet. Es preciso desarrollar estrategias de investigación que permitan detectar las interrelaciones y hacer predicciones.

\section{Fuentes consultadas}

- Abbasi, M. y otros (2012). Lessons Learned in Using Social Media for Disaster Relief. ASU Crisis Response Game, International Conference on Social Computing, Behavioral-Cultural Modeling, and Prediction. Maryland: College Park.

- Adam, G. (1999). “The Media and Complex Humanitarian Emergencies”. Humanitarian Exchange Magazine, núm. 13.

- Adams, W. (1986). "Whose Lives Count? TV Coverage of Natural Disasters". Journal of Communication, núm. 36, pp. 113-122.

- Aguirre,M.(1999). "Los medios periodísticos y el espectáculo humanitario”.En: Roberts, A. y otros. Los desafíos de la acción humanitaria: un balance. Barcelona: Icaria.

- Backstrom, L. (2010, Diciembre 14). “2010 Memology: Top Status Trends of the Year”. Extraída el 28/IX/2012 desde https://blog.facebook.com/blog.php?post=466369142130

- Bardin, L. (1986). El análisis de contenido. Madrid: Akal.

- Bratschi,G.(1995). Comunicando el desastre: Comunicación social preventiva y de emergencia en zonas sísmicas. Extensivo a otros desastres. Mendoza: Universidad Nacional de Cuyo. 
- Brown, P. y Minty, J. (2008). "Media Coverage and Charitable Giving after the 2004 Tsunami”. Southern Economic Journal, pp. 9-25.

- Carma International Global Media Analysts (2006). "Western media coverage of Humanitarian Disasters". Extraída el 28/IX/2012 desde http://www.comminit.com/ democracy-governance/node/243590

- Cate, F. (1996). From Massacres to Genocide: the Media, Public Policy and Humanitarian Crisis, Washington D. C.: Brookings Institution.

- Centre for Research on the Epidemiology of Disasters

_(2010). Annual Disasters Statistical Review. Extraída el 29/IX/2012 desde http://cip. management. dal.ca/publications/ADSR_2010.pdf

_(2009). Annual Disasters Statistical Review. Extraída el 28/IX/2012 desde http:// www.who.int/hac/techguidance/ems/annual_disaster_statistical_review_2009.pdf

- Ciancio, A. (2007). "Los medios de difusión y la acción humanitaria. Un nuevo marco para el debate". En Arcas, I. (ed.). Medios de comunicación y organizaciones humanitarias en la respuesta a las crisis. Madrid: Instituto de Estudios sobre Conflictos y Acción Humanitaria (IECAH).

- Coordinadora de ONG para el desarrollo - España (2007). Los medios de comunicación y las ONGD: situación actual y retos CONGDE. Madrid: CONGDE.

- Friedman, S. (2011). "Three Mile Island, Chernobyl, and Fukushima: An analysis of traditional and new media coverage of nuclear accidents and radiation". Bulletin of the Atomic Scientists, vol. 67, núm. 5, pp. 55-65.

- Galtung, J. y Ruge, M. (1965). “The Structure of Foreign News”. Journal of Peace Research, vol. 1, pp. 64-90.

- Gans, H. (1980). Deciding What's News: A study of CBS Evening News, NBC Nightly News, Newsweek and Time. Nueva York: Vintage Books.

- Gidley, R. (2007). "Eliminando los obstáculos: ¿Qué se interpone a las noticias humanitarias?". En Arcas, I. (ed.). Medios de comunicación y organizaciones humanitarias en la respuesta a las crisis. Madrid: Instituto de Estudios sobre Conflictos y Acción Humanitaria (IECAH).

- Gonzalo, P. (2011). “El Tsunami de Japón, mapas y redes sociales”. Extraída el 29/ IX/2012 desde http://www.periodismociudadano.com/category/colaboraciones/

- Gowing, N. (2001). Help or Hinderance in Conflict Prevention. Nueva York: Carnegie Commission on Preventing Deadly Conflict.

- Hens, M. (2007). "Una relación compleja: partiendo de la experiencia. Relatoría de la Mesa Redonda I", pp. 94-96. En Arcas. I. (ed.). Medios de comunicación y organizaciones humanitarias en la respuesta a las crisis. Madrid: Instituto de Estudios sobre Conflictos y Acción Humanitaria (IECAH).

- Holst,O.(1969).Content Analysis of the Social Sciences and Humanities. Massachusetts: Addison Wisely Publishing Company. 
- Jeong, Y. y Lee, S. (2010). “A Study on the News Values of International Disasters: Change of Determinants on News Coverage of International Disasters in the U.S. News Media". Artículo presentado en la reunión anual de la International Communication Association. Suntec City, Singapur.

- Jerez, A.; Sampedro, V. y López A. (2008). Del 0,7\% a la desobediencia civil, Política e Información del movimiento y las ONG de Desarrollo (1994-2000). Madrid: Centro de Investigaciones Sociológicas.

- Junk J. y Blatter, J. (2010). “Transnational Attention, Domestic Agenda-Setting and International Agreement: Modeling Necessary and Sufficient Conditions for Media-Driven Humanitarian Interventions". Discussion Paper SP IV 2010-301. Wissenschaftszentrum Berlin für Sozialforschung.

- Kremer, P. (2007). "Optimizar las relaciones entre los medios de comunicación y los actores humanitarios". En Arcas, I. (ed.). Medios de comunicación y organizaciones humanitarias en la respuesta a las crisis. Madrid: Instituto de Estudios sobre Conflictos y Acción Humanitaria (IECAH).

- Krippendorff, K. (1990). Metodología de análisis de contenido: teoría y práctica. Barcelona: Paidós.

- Martín Vivaldi, G. (1993). Géneros Periodísticos: reportaje, crónica, artículo. Madrid: Paraninfo.

- Mendiluce, J. (1997). Con rabia y esperanzas: retos y límites de la acción humanitaria. Madrid: Planeta.

- Meyer, P. (2002). Precision Journalism. A Reporter's Introduction to Social Science Methods. Maryland: Rowman and Littlefield.

- Minear, L.; Scott, C.y WEISS, T. (1996). The News Media, Civil War and Humanitarian Action. Londres: Lynne Rienner Publisher.

- Moeller, S.

_(2008). “Considering the Media's Framing and Agenda - Setting Roles in States' Responsiveness to Natural Crises and Disasters". Artículo presentado en el World Bank / Harvard Kennedy School Workshop on The Role of the News Media in the Governance Agenda. Massachusetts.

_(2006). "Regarding the Pain of Others: Media, Bias and the Coverage of International Disasters”. Journal of International Affairs, vol. 59, núm. 2, pp. 173-196.

- Mullaney, M. (2012). "Optimizing Social Media in Humanitarian Crisis Responses". The Macalester Review, vol. 2.

- Muralidharan, S. y otros (2011). "Hope for Haiti: An analysis of Facebook and Twitter usage during the earthquake relief efforts". Public Relations Review, núm. 37, pp. 175-177.

- Nagar, S., y otros (2012). "Characterization of Social Media Response to Natural Disasters". 21st International Conference companion on World Wide Web, pp- 671674. Nueva York: ACM. 
- Nos Aldás, E. (2002). "Construyendo una comunicación para la paz y la cooperación al desarrollo". En Nos Aldás, E. (ed.). Medios periodísticos, cooperación y acción humanitaria, ¿Relaciones Imposibles? Barcelona: Icaria.

- O’Heffernan, P. (1991). Mass Media and American Foreign Policy. Connecticut: Ablex Publising Corporation

- Pelling. M.; Maskrey, A.; Ruiz, P. y Hall, L. (2004). La reducción de riesgos de desastres: un desafío para el desarrollo. Nueva York: PNUD.

- Portales, P. (1988). "Noticias y catástrofes”. Chasqui, núm. 28, pp. 64-67.

- Reiersgord, B. (2011). "Technology and Disaster: The Case of Haiti and the Rise of Text Message Relief Donations. Case-Specific Briefing Paper Humanitarian Assistance in Complex Emergencies". University of Denver.

- Roberts, A. (1999). "El papel de las cuestiones humanitarias en la política internacional de los años noventa". En Roberts, A. y otros. Los desafíos de la acción humanitaria: un balance. Barcelona: Icaria.

- Rogers, E. y Sood, R. (1981). "Mass Media Operation in a quick - onset Natural Disaster: Hurricane David in Dominica”. Natural Hazard Research, núm. 41.

- Roosens, C. (1999). "La acción humanitaria y el sistema internacional vigente”. En Domestici-Met, M. (ed.). Geopolítica y Ayuda Humanitaria. Bilbao: Universidad de Deusto.

- Rosenstiel, T. y Mitchell, A. (2012). You Tube \& News. Pew Research Report.

- Russia Today (2011, Abril 4). "Japan 'tsunami dog' Ban reunited with owner after surviving at sea”. Extraída el 28/IX/2012 desde http://www.youtube.com/ watch?v=kG8wZjKEnNQ

- Sahagún, F. (2004). De Gutenberg a Internet. La sociedad internacional de la información. Madrid: Fragua.

- Sampedro, V. (1997). Movimientos sociales. Debates sin mordaza. Desobediencia civil y servicio militar (1970-1996). Madrid: BOE - Centro de Estudios Constitucionales.

- Simon, A. (1997). “Television News and International Earthquake Relief”. Journal of Communication, vol. 47, núm. 3, pp. 82-93.

- $\quad$ Tinker, T.; Fouse, D. y Curre, D. (2009). "Expert round table on social media and risk communication during times of crisis: strategic challenges and opportunities". Extraída el 29/IX/2012 desde: http://www.apha.org/NR/rdonlyres/47910BED-3371-46B385C267EFB80D88F8/0/socialmedreport.pdf

- Tristán, R. (2007). "La respuesta de los medios a las crisis internacionales". En Arcas, I. (ed.). Medios de comunicación y organizaciones humanitarias en la respuesta a las crisis. Madrid: Instituto de Estudios sobre Conflictos y Acción Humanitaria (IECAH).

- Tuchman, G. (1983). La producción de la noticia. Estudio sobre la construcción de la realidad. Barcelona: Gustavo Gili. 
- Van Belle, D.

_(2008). "Agenda-Setting and Donor Responsiveness to Humanitarian crisis and Development Aid”. Artículo presentado en el World Bank / Harvard Kennedy School Workshop on The Role of the News Media in the Governance Agenda. Massachusetts.

_(2000). "New York Times and Network TV News Coverage of Foreign Disasters: The Significance of the Insignificant Variables". Journalism and Mass Communication Quarterly, vol. 77, núm. 1, pp. 50-70.

- Vieweg, S. (2010). "Microblogged Contributions to the Emergency Arena: Discovery, Interpretation and Implications". $C S C W$, Georgia.

- Wolf, M. (2000). La investigación de la comunicación de masas. Crítica y perspectiva. Barcelona: Paidós.

- Wu, D. (2000). "Systematic Determinants of International News Coverage: A Comparison of 38 Countries". Journal of Communication, vol. 50, núm. 2, pp. 110-130. 